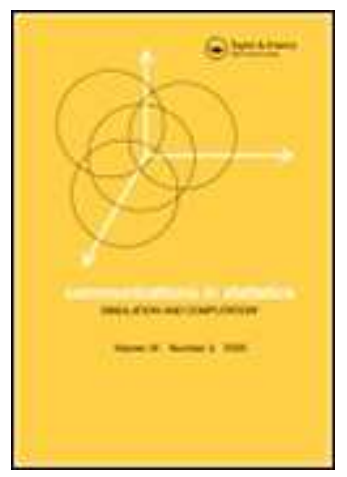

\title{
An Exponential Model for Damage Accumulation
}

\begin{tabular}{|c|c|}
\hline Journal: & Communications in Statistics - Simulation and Computation \\
\hline Manuscript ID: & LSSP-2008-0177.R1 \\
\hline Manuscript Type: & Original Paper \\
\hline $\begin{array}{r}\text { Date Submitted by the } \\
\text { Author: }\end{array}$ & 25-Aug-2008 \\
\hline Complete List of Authors: & $\begin{array}{l}\text { Balakrishnan, N.; McMaster University } \\
\text { Castillo, Enrique; University of Cantabria } \\
\text { Fern\'andez-Canteli, Alfonso; University of Oviedo } \\
\text { Kateri, Maria; University of Pireus, Statistics and Insurence Science }\end{array}$ \\
\hline Keywords: & $\begin{array}{l}\text { W } \backslash \text { "ohler field, damage accumulation, interpolation of fatigue } \\
\text { results, testing strategies, maximum likelihood method, constrained } \\
\text { maximum likelihood method }\end{array}$ \\
\hline Abstract: & $\begin{array}{l}\text { In this paper, we present } \\
\text { a general model for predicting the fatigue behavior for any stress } \\
\text { level and amplitude using the exponential model. Based on the } \\
\text { W\"ohler field for fixed stress level, a compatibility functional } \\
\text { equation enables us to derive the general model with eight } \\
\text { parameters. The problem of parameter estimation is then } \\
\text { discussed } \\
\text { and some methods are described. Some examples are finally } \\
\text { presented to illustrate the derived model and the proposed methods } \\
\text { of estimation. }\end{array}$ \\
\hline \multicolumn{2}{|c|}{$\begin{array}{l}\text { Note: The following files were submitted by the author for peer review, but cannot be converted } \\
\text { to PDF. You must view these files (e.g. movies) online. }\end{array}$} \\
\hline
\end{tabular}




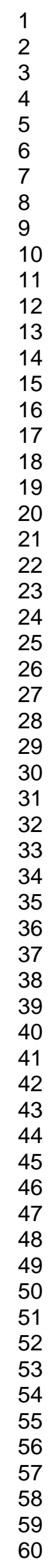

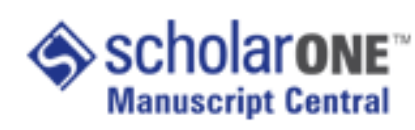
7 


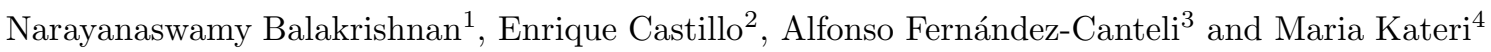 \\ ${ }^{1}$ Department of Mathematics and Statistics, McMaster University, Canada \\ 2 Department of Applied Mathematics and Computational Sciences, \\ University of Cantabria and University of Castilla-La Mancha, Spain \\ 3 Department of Construction and Manufacturing Engineering, \\ University of Oviedo, Spain \\ ${ }^{4}$ Department of Statistics and Insurance Science, University of Piraeus, Greece
}

\begin{abstract}
In this paper, we present a general model for predicting the fatigue behavior for any stress level and amplitude using the exponential model. Based on the Wöhler field for fixed stress level, a compatibility functional equation enables us to derive the general model with eight parameters. The problem of parameter estimation is then discussed and some methods are described. Some examples are finally presented to illustrate the derived model and the proposed methods of estimation.
\end{abstract}

Key Words: Wöhler field, damage accumulation, interpolation of fatigue results, testing strategies, exponential model, maximum likelihood method, constrained maximum likelihood method.

\title{
1 Introduction
}

In fatigue analysis that the stress ratio $R=\sigma_{\max } / \sigma_{\min }$ is an important factor, next only to the stress level $\left(\sigma_{\min }, \sigma_{\max }, \sigma_{\text {mean }}\right)$, which needs to be taken into account while dealing with fatigue behavior. This becomes particularly relevant while considering real stress histories instead of constant stress level situations. However, most of the existing laboratory test strategies and fatigue models are applicable for a given stress level but can not predict the fatigue behavior for different stress levels; see, for example, Coleman (1958), Bastenaire (1972), ASTM (1981), Spindel and Haibach (1981), Fernández-Canteli (1982), Castillo et al. (1985), Castillo and Galambos (1987), Castillo and Hadi (1995), Pascual and Meeker (1999), and Castillo and Fernández-Canteli (2001).

Though there are some models that take mean stress effects into account [see Conway and Sjodahlo (1991) and Dowling and Thangjitham (2000)], such simplified models are far from satisfactory. Castillo and Fernández-Canteli (2006) and Castillo et al. (2006) presented a general model that enables the prediction of the fatigue behavior based on two groups of tests run at two different stress levels. This was a significant development as other models did not facilitate this prediction and also that the required testing strategy for this model was very simple.

In this paper, we consider this general model for the case of the exponential distribution and describe the prediction of the fatigue behavior for any stress level and amplitude, and discuss some methods of estimation of the parameters underlying the model. In Section 2, we describe first the exponential parameter fatigue model. In Section 3, we describe how this model can be extrapolated to different test conditions, i.e., different pairs of $\sigma_{\max }$ and $\sigma_{\min }$, and then discuss the consequences of forcing the compatibility or independence of the results on the selected $\sigma_{\max }$ and $\sigma_{\min }$ test levels. In Section 4, we present a reparameterization of the model that is convenient for the estimation of the model parameters. In Section 5, we discuss some methods of estimation such as the maximum likelihood and constrained maximum likelihood methods. In Section 6, we present some examples to illustrate the derived model and the proposed methods of estimation. Finally, some concluding remarks are made in Section 7. 


\section{Derivation of the Fatigue Model}

Let us consider a series of tests with constant $\sigma_{\min }$ and variable $\sigma_{\max }$, but in each fatigue test we fix $\sigma_{\max }$ and repeat cycles in which $\sigma$ is varied from $\sigma_{\min }$ to $\sigma_{\max }$ until the failure of the specimen occurs. In other words, $\sigma_{\min }$ is constant for all tests, and different tests in the series can have identical or possibly different $\sigma_{\max }$. The resulting lifetimes $N$ (the number of cycles to failure) are observed. Since $\sigma_{\min }$ is held constant, we use as variables the stress ratio $R=\sigma_{\max } / \sigma_{\min }$ and $N$.

It is generally accepted that there are five variables initially involved in the fatigue problem: $P, N$, $N_{0}, R$ and $R_{0}$, where $P$ is the probability of fatigue failure of a piece when subjected to $N$ cycles, $N_{0}$ is the threshold value for $N$ corresponding to the minimum observable lifetime for any $R$, and $R_{0}$ is the endurance stress ratio limit below which fatigue failures can not occur. This means that there exists a relationship among the five initially independent variables, of the form

$$
r\left(N, N_{0}, R, R_{0}, P\right)=0,
$$

where $r(\cdot)$ is an initially unknown function. However, using the $\Pi$-Theorem [see, for example, Buckingham (1914, 1915a,b) and Castillo and Fernández-Canteli (2001)], these initial five variables can be reduced to three non-dimensional variables, viz., $N / N_{0}, R / R_{0}$ and $P$. Consequently, (1) can be written in terms of these three non-dimensional variables as

$$
r\left(N, N_{0}, R, R_{0}, P\right)=0 \quad \Longleftrightarrow \quad s\left(\frac{N}{N_{0}}, \frac{R}{R_{0}}, P\right)=0 ;
$$

since we are interested in $P$, we can express

$$
P=q\left(\frac{N}{N_{0}}, \frac{R}{R_{0}}\right),
$$

where $s(\cdot)$ and $q(\cdot)$ are functions to be determined. Thus, only the non-dimensional quotients $N / N_{0}$ and $R / R_{0}$ exert influence on the probability of failure $P$, and so either $N / N_{0}$ and $R / R_{0}$, or some monotone functions of them, say $h\left(N / N_{0}\right)$ and $g\left(R / R_{0}\right)$, need to be considered.

In this paper, we have chosen the $h$ and $g$ functions to be the logarithms of $N / N_{0}$ and $R / R_{0}$, respectively. For simplicity in notation, we denote throughout this paper

$$
N^{*}=\log \left(N / N_{0}\right), \quad R^{*}=\log \left(R / R_{0}\right), \quad N \geq N_{0}, \quad R \geq R_{0} .
$$

The selection of the exponential model is then based on the following important considerations:

1. Weakest link principle: This principle states that the fatigue lifetime of a longitudinal element is the minimum fatigue life of its constituent pieces. In other words, the selected family of distributions must hold (be valid) for different specimen lengths.

2. Limit behavior: To include the extreme case of the size of the supposed pieces constituting the element going to zero, or the number of pieces going to infinity, it is necessary for the distribution function family to be an asymptotic family; see Galambos (1987), Castillo (1988), Castillo et al. (2004), and Castillo et al. (2006).

3. Limited range: Experience shows that the selected non-dimensional variables, $N^{*}$ and $R^{*}$, have a finite lower end, which must coincide with the theoretical lower end of the selected cumulative distribution function (cdf).

4. Compatibility: In the $S$ - $N$ field, the $\operatorname{cdf} G\left(N^{*} ; R^{*}\right)$ of the lifetime given stress range should be compatible with the cdf of the stress range given lifetime $F\left(R^{*} ; N^{*}\right)$, i.e.,

$$
G\left(N^{*} ; R^{*}\right)=F\left(R^{*} ; N^{*}\right) .
$$


Though in standard tests $R^{*}$ is fixed and the associated random lifetime $N^{*}$ is determined, $R^{*}$ is interpreted here as the random stress that needs to be applied in order to produce failure at $N^{*}$.

It is important to note here that the exponential family satisfies all the conditions stated above.

\subsection{Compatibility}

The compatibility condition in (5) for the exponential model requires

$$
1-\exp \left[-\delta\left(N^{*}\right)\left(R^{*}-\lambda\left(N^{*}\right)\right)\right]=1-\exp \left[-\rho\left(R^{*}\right)\left(N^{*}-\eta\left(R^{*}\right)\right)\right],
$$

which leads to the functional equation

$$
R^{*} \delta\left(N^{*}\right)-\delta\left(N^{*}\right) \lambda\left(N^{*}\right)-\rho\left(R^{*}\right) N^{*}+\rho\left(R^{*}\right) \eta\left(R^{*}\right)=0
$$

whose solution is [see Castillo and Ruiz-Cobo (1992, p. 52) and Castillo et al. (2004, pp. 60-61)]

$$
\begin{aligned}
\rho\left(R^{*}\right) & =a R^{*}+b, \\
\eta\left(R^{*}\right) & =\frac{c R^{*}+d}{a R^{*}+b}, \\
\delta\left(N^{*}\right) & =a N^{*}-c, \\
\lambda\left(N^{*}\right) & =\frac{-b N^{*}+d}{a N^{*}-c} .
\end{aligned}
$$

This leads to the model

$$
G\left(N^{*} ; R^{*}\right)=F\left(R^{*} ; N^{*}\right)=1-\exp \left[\left(c-a N^{*}\right) R^{*}-b N^{*}+d\right],
$$

where $a, b, c$ and $d$ are arbitrary constants; now, upon reparameterizing and subsuming two parameters into $N_{0}$ and $R_{0}$, we obtain

$$
G\left(N^{*} ; R^{*}\right)=F\left(R^{*} ; N^{*}\right)=1-\exp \left[-\frac{R^{*} N^{*}-\lambda}{\delta}\right],
$$

where $\lambda$ and $\delta$ are some constants.

Once the model has been established in non-dimensional terms as in (13), as we need to recover the initial variables, we can reexpress the model in (13) as

$$
F(\log N ; \log R)=1-\exp \left\{-\left[\frac{(\log N-B)(\log R-C)-E}{D}\right]\right\}
$$

where $B=\log N_{0}, C=\log R_{0}$, and $E$ and $D$ are non-dimensional model parameters. Their physical meanings are as follows (see Figure 1 ):

$B$ : Threshold value of log-lifetime (logarithm of $N_{0}$ ),

$C$ : Endurance limit (logarithm of $R_{0}$ ),

$E$ : Parameter defining the position of the corresponding zero-percentile hyperbola,

$D$ : Scale factor. 


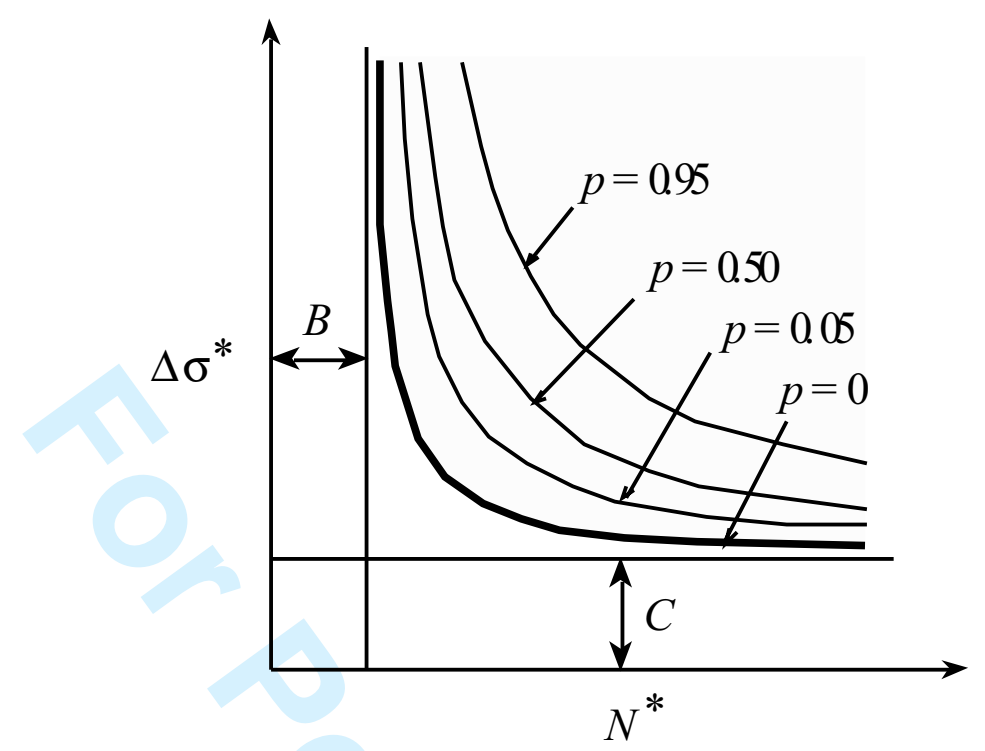

Figure 1: Percentiles curves representing the relationship between lifetime, $N^{*}$, and stress range, $\Delta \sigma^{*}$, in the $S$ - $N$ field for the fatigue model in (14).

The percentile curves are shown in Figure 1. The zero-percentile curve represents the minimum possible required number of cycles of fatigue failure for different values of $R$. All the remaining percentiles happen to be the positive branches of a hyperbola, i.e.,

$$
N^{*} R^{*}=E+D[-\log (1-p)],
$$

where $p \in[0,1]$ defines the percentile. For such curves, the minimum number of cycles to fatigue failure decreases with increasing $R^{*}$, which is in agreement with experimental results. The parameters $E$ and $D$ determine the $p$-percentile, and hence define a unique curve on the plot.

It can be seen from (13) that

$$
N^{*} R^{*} \sim \operatorname{Exp}(\lambda, \delta) \quad \Leftrightarrow \quad N^{*} \sim \operatorname{Exp}\left(\frac{E}{R^{*}}, \frac{D}{R^{*}}\right),
$$

for given $R^{*}$, where $\operatorname{Exp}(\lambda, \delta)$ denotes a two-parameter exponential distribution with $\lambda$ as the threshold parameter and $\delta$ as the scale parameter. It is of interest to note that (15) has a non-dimensional form and reveals that the probability of failure of a piece subject to a stress ratio $R^{*}$ during $N^{*}$ cycles depends only on the product $N^{*} R^{*}$. Thus, $V=N^{*} R^{*}$ is useful for comparing fatigue strength at different stress levels that are maintained constant, and can be considered as a normalizing variable.

\section{Physical and compatibility considerations}

Since for each constant $\sigma_{m i n}$, a model as in (14) is obtained, we can consider its parameters $B, C, D$ and $E$ to be functions of $\sigma_{\min }$. Figure 2(a) shows the Wöhler curves for two groups of tests run at constant $\sigma_{\min }=0.8$ and at constant $\sigma_{\min }=0.4$. Note that larger values of $\sigma_{\min }$ lead to lower percentile curves. If we consider the Wöhler curves for two groups of tests run at constant $\sigma_{\max }$, say $\sigma_{\max }=1.5$, we obtain the model in (14) once again and a set of percentiles, for example $\{0.01,0.05,0.5,0.95,0.99\}$, are shown 
in Figure 2(b). However, if the tests are run at constant $\sigma_{\max }=1$, the resulting percentiles change as displayed in the same figure. Note that larger values of $\sigma_{\max }$ lead to lower percentile curves.

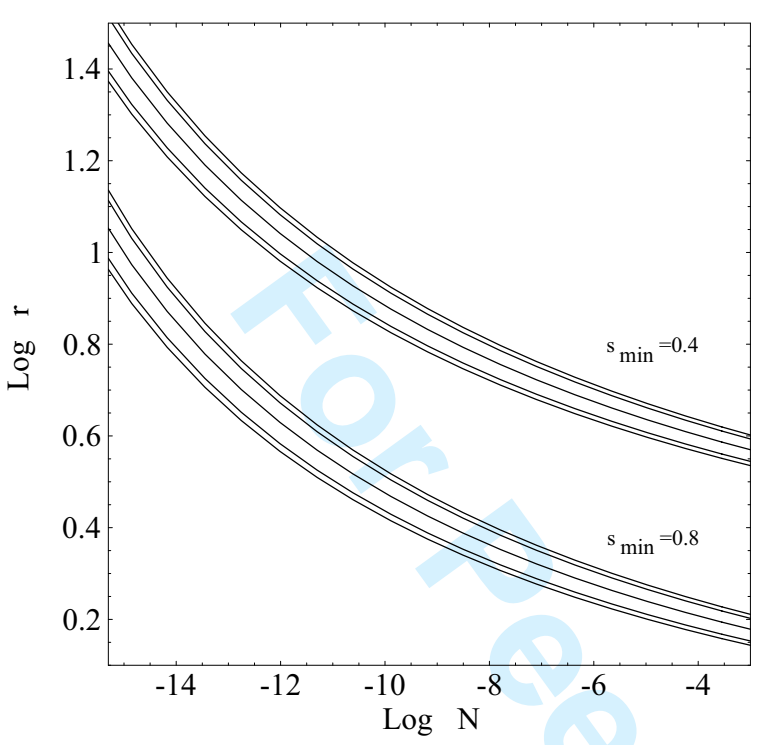

(a)

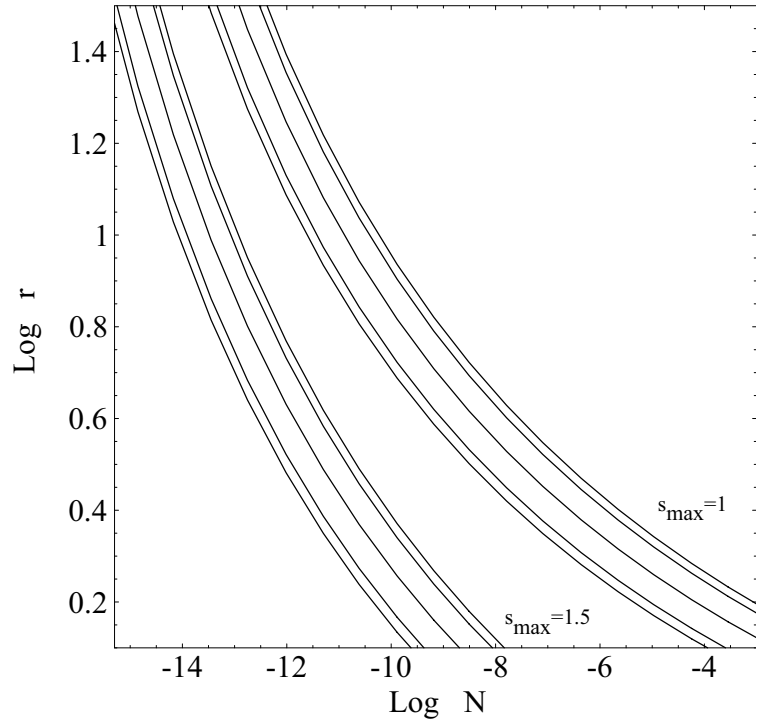

(b)

Figure 2: Wöhler curves for percentiles 0.01, 0.05, 0.5, 0.95, 0.99: (a) for constant $\sigma_{\min }=0.4$ and $\sigma_{\min }=$ 0.8 , and (b) for constant $\sigma_{\max }=1$ and $\sigma_{\max }=1.5$.

An important property to be observed is that the same percentile curves never intersect if they are associated with two different $\sigma_{\max }$ or with two different $\sigma_{\min }$, but they intersect if one set of percentiles is associated with constant $\sigma_{\max }$ and another with constant $\sigma_{\min }$ as shown in Figure 3, where the four sets of percentiles have been plotted together, with dashed lines corresponding to Wöhler curves for constant $\sigma_{\min }$ and continuous lines corresponding to Wöhler curves for constant $\sigma_{\max }$. Note that the line joining the intersections of associated percentiles for one constant $\sigma_{\max }$ and one constant $\sigma_{\min }$ must be straight and horizontal since both fields, i.e., the associated cdf's, must coincide. This is a very strong condition indeed and it assists greatly in deriving a simple model which is able to deal with different stress levels. Though in Figure 3 we have these four sets, we realize that there is an infinite set of them. More precisely, two families of S-N curves exist, one for different constant values of $\sigma_{\max }$ and another for different constant values of $\sigma_{\min }$.

In this paper, we consider the case when the test experiments are conducted for constant $\sigma_{\max }=\sigma_{M}$ and constant $\sigma_{\min }=\sigma_{m}$. As mentioned above, the model in (14) is valid only if all tests are conducted at the same stress level, and in particular, for given values of $\sigma_{\max } \equiv \sigma_{M}$ or $\sigma_{\min } \equiv \sigma_{m}$. Thus, if two sample data do not coincide at either their $\sigma_{M}$ or $\sigma_{m}$, the model can not be applied. Since there are two different cases, we must consider two fatigue models of the type in (14), i.e., we initially have two sets of parameters $B_{m}, C_{m}, D_{m}, E_{m}$ and $B_{M}, C_{M}, D_{M}, E_{M}$, where the subindices $m$ and $M$ have been used for the cases of constant $\sigma_{\min }=\sigma_{m}$ and constant $\sigma_{\max }=\sigma_{M}$, respectively. However, for the same stress amplitude and level, i.e., when both $\sigma_{M}$ and $\sigma_{m}$ coincide, for the compatibility condition to hold, we must have the same model for both cases, i.e., for all $N$ we must have

$$
\frac{\left(\log N-B_{m}\left(\sigma_{m}\right)\right)\left(\log R-C_{m}\left(\sigma_{m}\right)\right)-E_{m}\left(\sigma_{m}\right)}{D_{m}\left(\sigma_{m}\right)}=\frac{\left(\log N-B_{M}\left(\sigma_{M}\right)\right)\left(\log R-C_{M}\left(\sigma_{M}\right)-E_{M}\left(\sigma_{M}\right)\right.}{D_{M}\left(\sigma_{M}\right)} .
$$




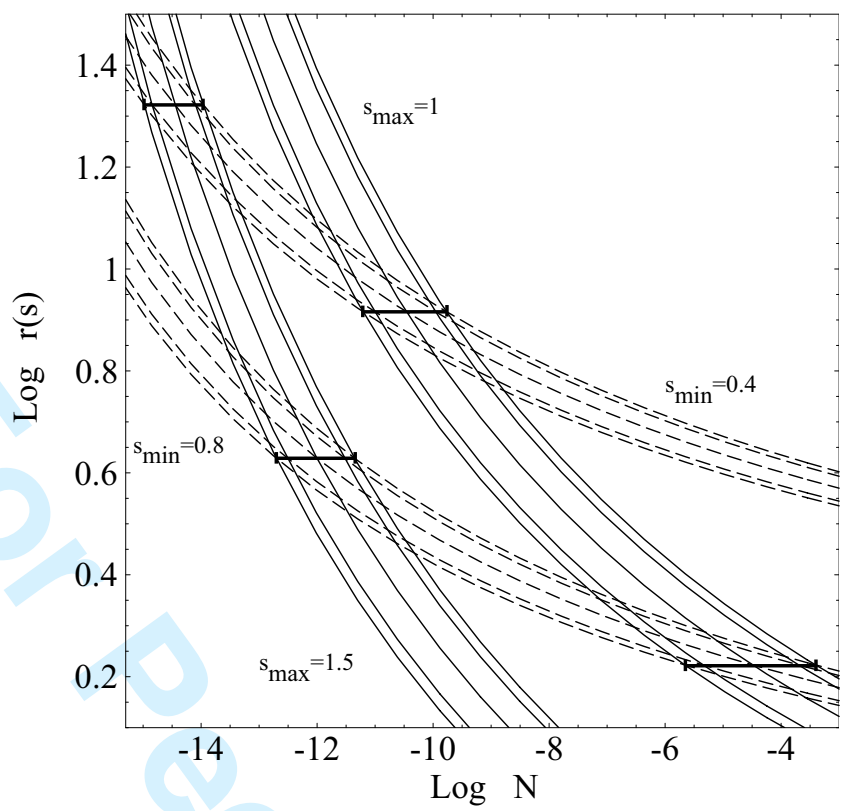

Figure 3: Wöhler curves for percentiles $\{0.01,0.05,0.5,0.95,0.99\}$ for $\sigma_{\max }=1$ and $\sigma_{\max }=1.5$, and $\sigma_{m i n}=0.4$ and $\sigma_{\min }=0.8$. Dashed lines refer to Wöhler curves for constant $\sigma_{m i n}$, and continuous lines refer to Wöhler curves for constant $\sigma_{\max }$.

For the functional equation in (16) to be satisfied for any $N, \sigma_{m}$ and $\sigma_{M}$, both models must have the same parameters. Rewriting now the model in (16) as

$$
\frac{\log N-\left[B_{m}\left(\sigma_{m}\right)+\frac{E_{m}\left(\sigma_{m}\right)}{\log R-C_{m}\left(\sigma_{m}\right)}\right]}{\frac{D_{m}\left(\sigma_{m}\right)}{\log R-C_{m}\left(\sigma_{m}\right)}}=\frac{\log N-\left[B_{M}\left(\sigma_{M}\right)+\frac{E_{M}\left(\sigma_{M}\right)}{\log R-C_{M}\left(\sigma_{M}\right)}\right]}{\frac{D_{M}\left(\sigma_{M}\right)}{\log R-C_{M}\left(\sigma_{M}\right)}} \forall N
$$

and forcing the exponential parameters to coincide, we obtain

$$
\begin{aligned}
\frac{D_{m}\left(\sigma_{m}\right)}{D_{M}\left(\sigma_{M}\right)} & =\frac{\log \sigma_{M}-\log \sigma_{m}-C_{m}\left(\sigma_{m}\right)}{\log \sigma_{M}-\log \sigma_{m}-C_{M}\left(\sigma_{M}\right)} \forall \sigma_{m}, \sigma_{M} \\
B_{M}\left(\sigma_{M}\right) & =B_{m}\left(\sigma_{m}\right)-\frac{E_{M}\left(\sigma_{M}\right)}{\log \sigma_{M}-\log \sigma_{m}-C_{M}\left(\sigma_{M}\right)}+\frac{E_{m}\left(\sigma_{m}\right)}{\log \sigma_{M}-\log \sigma_{m}-C_{m}\left(\sigma_{m}\right)} \quad \forall \sigma_{m}, \sigma_{M} .
\end{aligned}
$$

The functional equations in (18) and (19) deserve a careful attention to get a deeper understanding of our problem; they are not simple equalities, but each a full collection of equalities as they hold for any feasible pair $\sigma_{m}, \sigma_{M}$. Eqs. (18) and (19), considered as functions of $\sigma_{m}$, must be independent of $\sigma_{M}$. This simple but powerful condition allows us to derive the structure of the functions $D_{m}\left(\sigma_{m}\right), D_{M}\left(\sigma_{M}\right)$ and $B_{M}\left(\sigma_{M}\right)$. The complete derivation, as given by Castillo et al. (2006), leads to the final model as

$$
\begin{aligned}
F\left(N ; \sigma_{m}, \sigma_{M}\right)= & 1-\exp \left\{-\left[C_{0}+C_{1} \log N+C_{2} \log \sigma_{m}+C_{3} \log \sigma_{M}+C_{4} \log N \log \sigma_{m}\right.\right. \\
& \left.\left.+C_{5} \log N \log \sigma_{M}+C_{6} \log \sigma_{m} \log \sigma_{M}+C_{7} \log N \log \sigma_{m} \log \sigma_{M}\right]\right\}
\end{aligned}
$$




$$
\begin{aligned}
= & 1-\exp \left\{-\left[\left(C_{0}+C_{2} \log \sigma_{m}+C_{3} \log \sigma_{M}+C_{6} \log \sigma_{m} \log \sigma_{M}\right)\right.\right. \\
& \left.\left.+\log N\left(C_{1}+C_{4} \log \sigma_{m}+C_{5} \log \sigma_{M}+C_{7} \log \sigma_{m} \log \sigma_{M}\right)\right]\right\} .
\end{aligned}
$$

Observe that this model involves eight parameters $C_{i}(i=0,1, \ldots, 7)$.

\subsection{Physical conditions}

For the model to be physically feasible, the constants must satisfy some constraints. In particular:

- The cdf in (21) must be increasing in $\log N$;

- The cdf in (21) must be non-increasing in $\sigma_{m}$;

- The cdf in (21) must be non-decreasing in $\sigma_{M}$;

- The curvature of the zero-percentile of $(\log N, \log R)$ for constant $\sigma_{m i n}$ must be non-negative;

- The curvature of the zero-percentile of $(\log N, \log R)$ for constant $\sigma_{\max }$ must be non-negative.

\subsection{Resulting models}

In summary, we can obtain some final set of feasible submodels of (21) as follows:

Linear Model: The simplest model with no asymptotes is given by

$$
\begin{aligned}
& F\left(N ; \sigma_{m}, \sigma_{M}\right)=1-\exp \left\{-\left[C_{0}+C_{1} \log N+C_{2} \log \sigma_{m}+C_{3} \log \sigma_{M}\right]\right\}, \\
& C_{1}, C_{2} \leq 0, C_{3} \leq 0 \text {, }
\end{aligned}
$$

which in log-log scale leads to a Wöhler field made of straight lines.

Model with asymptotes independent on $\sigma_{m}$ and $\sigma_{M}$ : The model with $\log N$ asymptotes independent on $\sigma_{m}$ and $\sigma_{M}$ is given by

$$
\begin{array}{r}
F\left(N ; \sigma_{m}, \sigma_{M}\right)=1-\exp \left\{-\left[C_{0}+C_{2} \log \sigma_{m}+C_{3} \log \sigma_{M}+C_{4} \log N \log \sigma_{m}+C_{5} \log N \log \sigma_{M}\right]\right\}, \\
C_{2}, C_{4} \leq 0, C_{3}, C_{5} \geq 0, C_{4}+C_{5} \geq 0,
\end{array}
$$

which is obtained when $C_{1}=C_{6}=C_{7}=0$.

The $\log N$ asymptotes are $\log N=-\frac{C_{3}}{C_{5}}$ for the case of constant $\sigma_{m i n}$, and $\log N=-\frac{C_{2}}{C_{4}}$ for the case of constant $\sigma_{\max }$, and the $\log R$ asymptotes are $\log R=-\left(C_{4}+C_{5}\right) \log \sigma_{m} / C_{5}$ for the case of constant $\sigma_{\min }$, and $\log R=\left(C_{4}+C_{5}\right) \log \sigma_{M} / C_{4}$ for the case of constant $\sigma_{\max }$.

Model with fixed asymptotes: The model with $\log R$ asymptotes independent on $\sigma_{m}$ and $\sigma_{M}$ is given by

$$
\begin{aligned}
F\left(N ; \sigma_{m}, \sigma_{M}\right)= & 1-\exp \left\{-\left[C_{0}+C_{1} \log N+C_{2} \log \sigma_{m}+C_{3} \log \sigma_{M}+C_{4} \log N\left(\log \sigma_{m}-\log \sigma_{M}\right)\right.\right. \\
& \left.\left.+C_{6} \log \sigma_{m} \log \sigma_{M}\right]\right\},
\end{aligned}
$$

which is obtained when $C_{7}=0$ and $C_{4}+C_{5}=0$. The $\log R$ asymptotes are $\log R=-C_{1} / C_{5}$ for the case of constant $\sigma_{\min }$, and $\log R=C_{1} / C_{4}$ for the case of constant $\sigma_{\max }$.

The $\log N$ asymptotes are $\log N=-\frac{C_{3}+\log \sigma_{M} C_{6}}{C_{5}}$ for the case of constant $\sigma_{\text {min }}$, and $\log N=$ $-\frac{C_{2}+\log \sigma_{M} C_{6}}{C_{4}}$ for the case of constant $\sigma_{m a x}$, so that they are constant if, in addition, $C_{6}=0$. 
General Model: The general model with $\log R$ and $\log N$ asymptotes dependent on $\sigma_{m}$ and $\sigma_{M}$ is given by

$$
\begin{aligned}
F\left(N ; \sigma_{m}, \sigma_{M}\right)= & 1-\exp \left\{-\left[C_{0}+C_{1} \log N+C_{2} \log \sigma_{m}+C_{3} \log \sigma_{M}+C_{4} \log N \log \sigma_{m}\right.\right. \\
& \left.\left.+C_{5} \log N \log \sigma_{M}+C_{6} \log \sigma_{m} \log \sigma_{M}+C_{7} \log N \log \sigma_{m} \log \sigma_{M}\right]\right\},
\end{aligned}
$$

subject to adequate constraints on the parameters.

These constraints are very important in order to have a physically meaningful model.

\subsection{Testing strategies}

The aim of any testing strategy is to estimate the model parameters in (21). For this purpose, a testing strategy involving one single group of tests with constant $\sigma_{\max }$ or one single group with constant $\sigma_{\min }$ or two groups one with constant $\sigma_{\max }$ and one with constant $\sigma_{\min }$ are not sufficient, because of the linear combination of the parameters that are involved in the model. In contrast, two groups of tests one with constant $\sigma_{\max }$ and one with constant $\sigma_{\min }$ are sufficient for estimating the parameters. Many other alternatives are also possible by combining different constant (more than one) levels of $\sigma_{\max }$ or constant $\sigma_{\min }$. A very efficient testing strategy, in particular, consists of selecting two different values of $\sigma_{\max }$ and two different values of $\sigma_{\min }$ and combine them to obtain 4 groups of tests, for which several specimens must be tested.

\section{Convenient Reparameterized Model}

In order to estimate the cumulative distribution function, the model in (21) is reparameterized in such a way that it can be viewed as a two-parameter exponential distribution in which case the required estimation becomes simple. So, the model in (21) is expressed as

$$
F\left(\log N ; \sigma_{m_{i}}, \sigma_{M_{j}}\right)=1-\exp \left\{-\left[C_{0 i j}+C_{1 i j} \log N\right]\right\},
$$

for $i, j=1,2$, where

$$
C_{0 i j}=C_{0}+C_{2} \log \sigma_{m_{i}}+C_{3} \log \sigma_{M_{j}}+C_{6} \log \sigma_{m_{i}} \log \sigma_{M_{j}}
$$

and

$$
C_{1 i j}=C_{1}+C_{4} \log \sigma_{m_{i}}+C_{5} \log \sigma_{M_{j}}+C_{7} \log \sigma_{m_{i}} \log \sigma_{M} M_{j} .
$$

We then recognize that $\log N$, with $\log N \geq-C_{0 i j} / C_{1 i j}$ and $C_{1 i j}>0$, follows a two-parameter exponential distribution with $\gamma_{i j}=-C_{0 i j} / C_{1 i j}$ and $\beta_{i j}=1 / C_{1 i j}$ as the location and scale parameters, respectively. We now use the expression in (26) for the purpose of estimation. Once the parameters $C_{0 i j}$ and $C_{1 i j}$ are all estimated, the expressions in (27) and (28) evaluated at these estimates for the four conditions produced by two different values for $\sigma_{m}\left(\sigma_{m_{1}}, \sigma_{m_{2}}\right)$ and $\sigma_{M}\left(\sigma_{M_{1}}, \sigma_{M_{2}}\right)$, provide a system of eight equations, using which the estimates of the eight initial parameters $C_{i}(i=0,1, \ldots, 7)$ can be achieved. Solving this system of eight equations, we get

$$
\begin{aligned}
C_{7} & =\frac{C_{111}-C_{112}-C_{121}+C_{122}}{\left(\log \sigma_{m_{1}}-\log \sigma_{m_{2}}\right)\left(\log \sigma_{M_{1}}-\log \sigma_{M_{2}}\right)}, \\
C_{5} & =\frac{C_{111}-C_{112}}{\log \sigma_{M_{1}}-\log \sigma_{M_{2}}}-C_{7} \log \sigma_{m_{1}}, \\
C_{4} & =\frac{C_{111}-C_{121}}{\log \sigma_{m_{1}}-\log \sigma_{m_{2}}}-C_{7} \log \sigma_{M_{1}}, \\
C_{1} & =C_{111}-C_{4} \log \sigma_{m_{1}}-C_{5} \log \sigma_{M_{1}}-C_{7} \log \sigma_{m_{1}} \log \sigma_{M_{1}},
\end{aligned}
$$




\section{$5 \quad$ Parameter Estimation}

In this section, we describe two methods of estimating the parameters of the model.

1. Method 1 (based on the maximum likelihood on 4 stress level tests and least-squares) From the model in (26), the sample log-likelihood, taking the run-outs into account, turns out to be

$$
L=-\sum_{i, j=1}^{2} \sum_{k=1}^{n_{i j}}\left(C_{0 i j}+C_{1 i j} \log N_{i j k}\right)+\sum_{i, j=1}^{2} n_{i j}^{*} \log C_{1 i j},
$$

where $N_{i j k}, n_{i j}$ and $n_{i j}^{*}$ are the lifetime (or the censored value) of the specimen $k$, the total number of specimens, and the number of uncensored specimens, respectively, tested at stress levels $\sigma_{m_{i}}$ and $\sigma_{M_{j}}$. Then, by standard results for the two-parameter exponential distribution, we have the maximum likelihood estimates (MLEs) to be

$$
\hat{C}_{1 i j}=\frac{n_{i j}^{*}}{\sum_{k=1}^{n_{i j}}\left[\log N_{i j k}-\log N_{\min _{i j}}\right]}
$$

and

$$
\hat{C}_{0 i j}=-\log N_{\min _{i j}} \hat{C}_{1 i j}, \quad i, j=1,2,
$$

where $N_{\min _{i j}}=\min \left(N_{i j 1}, N_{i j 2}, \ldots, N_{i j k_{i j}}\right)$ denotes the smallest lifetime observed for stress levels $\sigma_{m_{i}}$ and $\sigma_{M_{j}}$. The MLEs of the initial parameters in (21) are then obtained by replacing in Eqs. (29) - (36) $C_{0 i j}$ and $C_{1 i j}$ by their MLEs in (39) and (38), respectively. However, if one uses any of the submodels presented in Section 3.2, then there will be more equations than unknown parameters. In this case, we propose to get the estimates of the initial parameters by minimizing the sum of squares based on the MLEs $C_{0 i j}$ and $C_{1 i j}$ given by

$$
\begin{aligned}
Q=\sum_{i, j} & {\left[\left\{C_{0 i j}-\left(C_{0}+C_{2} \log \sigma_{m_{i}}+C_{3} \log \sigma_{M_{j}}+C_{6} \log \sigma_{m_{i}} \log \sigma_{M_{j}}\right)\right\}^{2}\right.} \\
+ & \left.\left\{C_{1 i j}-\left(C_{1}+C_{4} \log \sigma_{m_{i}}+C_{5} \log \sigma_{M_{j}}+C_{7} \log \sigma_{m_{i}} \log \sigma_{M} M_{j}\right)\right\}^{2}\right],
\end{aligned}
$$

wherein the parameters constraints can also be included in the optimization process, if desired.

In this manner, we may avoid the constrained maximum likelihood estimation method used so far for these types of problems; see, for example, Castillo et al. (2006). At this stage, we can test whether the physical constraints of the model are satisfied by the estimates determined by the above method. In case they are, the computed estimates are indeed the desired MLEs. If not, the unconstrained MLEs do not belong to the restricted parameter space and in this case the constrained MLEs need to be computed by a numerical method. To this end, existing software such as GAMS enables not only to solve the problem but also to perform a sensitivity analysis; for details, see Castillo et al. (2001, 2004) and Conejo et al. (2006)). 
2. Method 2 (based on constrained maximum likelihood) - In this case, we maximize the log-likelihood function

$$
\begin{aligned}
L= & -\sum_{i, j=1}^{2} \sum_{k=1}^{n_{i j}}\left(C_{0}+C_{2} \log \sigma_{m_{i}}+C_{3} \log \sigma_{M_{j}}+C_{6} \log \sigma_{m_{i}} \log \sigma_{M_{j}}\right. \\
& \left.+\left(C_{1}+C_{4} \log \sigma_{m_{i}}+C_{5} \log \sigma_{M_{j}}+C_{7} \log \sigma_{m_{i}} \log \sigma_{M} M_{j}\right) \log N_{i j k}\right) \\
& +\sum_{i, j=1}^{2} n_{i j}^{*} \log C_{1}+C_{4} \log \sigma_{m_{i}}+C_{5} \log \sigma_{M_{j}}+C_{7} \log \sigma_{m_{i}} \log \sigma_{M} M_{j}
\end{aligned}
$$

with respect to the parameters $C_{i}(i=0,1, \ldots, 7)$ subject to the constraints

$$
C_{0}+C_{2} \log \sigma_{m}+C_{3} \log \sigma_{M}+C_{4} \log N_{i j k} \log \sigma_{m}+C_{5} \log N_{i j k} \log \sigma_{M} \geq 0, \quad \forall i, j, k,
$$

and additional constraints to be satisfied by the parameters for the different models as presented earlier in Section 3.2.

\section{Illustrative Examples}

The proposed estimation methods are illustrated in this section with some data sets. For the set of parameter values $C_{0}=-407.905, C_{1}=0, C_{2}=-1.272, C_{3}=53.132, C_{4}=-1.755, C_{5}=2.107, C_{6}=$ $0, C_{7}=0$ and for each of the four combinations of the two values of $\sigma_{\min }\left(\sigma_{\min }=510,714\right)$ and two values of $\sigma_{\max }\left(\sigma_{\max }=1054,1190\right)$, two samples were simulated, one of small size $(n=7)$ and another of moderate size $(n=20)$. The $\log N$ values of these samples are presented in Table 1 .

The model parameters were then estimated by using the two methods described in Section 5 .

Method 1: The MLEs $\hat{C}_{0 i j}$ and $\hat{C}_{1 i j}(i, j=1,2)$ and the corresponding MLEs $\hat{C}_{i}(i=0,1, \ldots, 7)$ estimates are provided in Columns 3 and 4 of Table 2 as "ML unconstrained estimates", for the cases when $n=7$ and 20 without runouts. The true values of the parameters of the model, viz., $C_{0 i j}, C_{1 i j}(i, j=1,2)$ and $C_{i}(i=0,1, \ldots 7)$, are also presented in Column 2 for the purpose of comparison. The parameters $C_{0 i j}$ and $C_{1 i j}$ are the ones that are crucial for modelling and prediction purposes. We observe that even though the estimation of the initial parameters is not accurate, the estimates of the parameters $C_{0 i j}$ and $C_{1 i j}$ parameters (which are functions of them) are quite good.

Further, in order to illustrate the influence of run-outs, we also considered additional threshold values. For each condition, we considered the corresponding 90-th percentile $Q_{90}\left(\sigma_{m}, \sigma_{M}\right)$ as threshold value, which are $Q_{90}(510,1054)=12.97520, Q_{90}(510,1190)=10.5213, Q_{90}(714,1054)=15.5570$ and $Q_{90}(714,1190)=12.4807$. Thus, for the samples presented in Table 1, we observe the number of run-outs to be $1,1,1,1$ for the case when $n=7$, and 3,3,3,2 for the case when $n=20$. The estimates of the parameters of the original model as well as those of the reparameterized model are presented in Columns 5 and 6 of Table 2. Moreover, the quartiles of the true parameter values and the estimated values are presented in Table 3 , while the cdf's as well as their estimates are presented in Figure 4. From the values of $\hat{C}_{0 i j}$ and $\hat{C}_{1 i j}$, as well as from the plots, it is clear that increasing sample size results in more precision while the presence of run-outs result in loss of precision in the estimation.

Method 2: We fitted the model in (23) using the constrained maximum likelihood method described in Section 5. The parameter estimates so obtained are presented in Table 2 as "Constrained estimates". It is clear that the fitted values of the original $C_{i}$ parameters are better than those 
Table 1: Simulated $\log N$ ordered values for the four different conditions of (a) 7 and (b) 20 specimens at each condition.

(a)

\begin{tabular}{l|cccccccc}
\hline Condition & \multicolumn{7}{|c}{ Simulated $\log N$ values } \\
\hline $\begin{array}{l}\sigma_{m}=510 \\
\sigma_{M}=1054\end{array}$ & 12.4235 & 12.4396 & 12.5503 & 12.6968 & 12.8483 & 12.854 & 13.0437 \\
\hline$\sigma_{m}=510$ & & & & & & & \\
$\sigma_{M}=1190$ & 9.95172 & 9.98658 & 10.0196 & 10.1651 & 10.2444 & 10.2453 & 10.7026 \\
\hline$\sigma_{m}=714$ & & & & & & & \\
$\sigma_{M}=1054$ & 14.8401 & 14.8985 & 14.9619 & 14.9907 & 15.3109 & 15.3237 & 15.7461 \\
\hline $\begin{array}{l}\sigma_{m}=714 \\
\sigma_{M}=1190\end{array}$ & 11.8056 & 11.8549 & 11.9707 & 11.9759 & 12.041 & 12.3971 & 12.7405 \\
\hline
\end{tabular}

(b)

\begin{tabular}{l|rrrrrrrrrr}
\hline Condition & \multicolumn{10}{|c}{ Simulated $\log N$ values } \\
\hline$\sigma_{m}=510$ & 12.3879 & 12.3945 & 12.4266 & 12.4364 & 12.4759 & 12.494 & 12.4955 & 12.5004 & 12.5329 & 12.6312 \\
$\sigma_{M}=1054$ & 12.643 & 12.7081 & 12.7257 & 12.7924 & 12.7981 & 12.8534 & 12.8892 & 12.9967 & 13.0073 & 13.0074 \\
\hline$\sigma_{m}=510$ & 9.96423 & 9.98116 & 9.99038 & 10.016 & 10.0338 & 10.0425 & 10.0432 & 10.0482 & 10.0941 & 10.0995 \\
$\sigma_{M}=1190$ & 10.1064 & 10.1385 & 10.1428 & 10.2108 & 10.211 & 10.2116 & 10.2846 & 10.8629 & 10.8929 & 11.0735 \\
\hline$\sigma_{m}=714$ & 14.8585 & 14.8848 & 14.8892 & 14.8893 & 14.8984 & 14.8998 & 14.9122 & 14.9212 & 14.9253 & 15.0038 \\
$\sigma_{M}=1054$ & 15.0649 & 15.0831 & 15.1102 & 15.2873 & 15.384 & 15.3964 & 15.5167 & 15.6858 & 15.8732 & 15.9394 \\
\hline$\sigma_{m}=714$ & 11.8027 & 11.8065 & 11.8129 & 11.8224 & 11.8371 & 11.8609 & 11.9272 & 11.9326 & 11.9428 & 11.9901 \\
$\sigma_{M}=1190$ & 12.0414 & 12.0539 & 12.0895 & 12.090 & 12.1639 & 12.2018 & 12.2985 & 12.2991 & 12.8947 & 13.1203 \\
\hline
\end{tabular}

obtained by Method 1. The quantile estimates are also presented in Table 3 as "Constrained estimates" which are also better than those obtained by Method 1 .

We thus observe that the two methods of estimation proposed in Section 5 offer convenient methods of modeling the fatigue behavior, and that Method 1 presents a simpler alternative to the computationally involved constrained maximum likelihood method.

\section{Conclusions}

In this paper, we have presented a general model for the prediction of the fatigue behavior for any stress level and amplitude using an exponential model and discuss some important considerations such as weakest link principle, limit behavior, limited range, and compatibility. Using the compatibility condition, an exponential model is obtained with some non-dimensional model parameters whose physical interpretations are given in terms of threshold value of log-lifetime, endurance limit, parameter defining the position of the corresponding zero-percentile hyperbola, and scale factor. Next, based on physical and compatibility considerations and on the Wöhler field for fixed stress level, a compatibility functional equation yields a general exponential model with eight parameters. We have then reparametrized this general model into a two-parameter exponential distribution and have explained how the estimation of its parameters can be used to set-up a system of eight equations for the estimation of the eight parameters of the original model. For the estimation method, we have described two methods, one based on the maximum likelihood on four stress level tests and least-squares, and the other based on the constrained 
Table 2: True and estimated values of the parameters, based on the two samples in Table 1, with and without run-outs.

\begin{tabular}{|c|c|c|c|c|c|}
\hline \multirow{2}{*}{\multicolumn{2}{|c|}{ Parameter True value }} & \multirow{2}{*}{\multicolumn{2}{|c|}{$\begin{array}{c}\text { Estimated value }^{1} \\
(n=7) \quad(n=20)\end{array}$}} & \multicolumn{2}{|c|}{ Estimated value $^{2}$} \\
\hline & & & & $(n=7)$ & $(n=20)$ \\
\hline & & \multicolumn{4}{|c|}{ ML unconstrained estimates } \\
\hline$C_{011}$ & -46.018 & -45.972 & -45.554 & -39.404 & -38.721 \\
\hline$C_{111}$ & 3.724 & 3.700 & 3.677 & 3.172 & 3.126 \\
\hline$C_{012}$ & -39.570 & -42.140 & -38.593 & -36.120 & -32.804 \\
\hline$C_{112}$ & 3.980 & 4.234 & 3.873 & 3.630 & 3.292 \\
\hline$C_{021}$ & -46.446 & -47.405 & -47.512 & -40.633 & -40.386 \\
\hline$C_{121}$ & 3.134 & 3.194 & 3.198 & 2.738 & 2.718 \\
\hline$C_{022}$ & -39.998 & -38.499 & -39.767 & -32.999 & -35.791 \\
\hline$C_{122}$ & 3.389 & 3.261 & 3.369 & 2.795 & 3.032 \\
\hline$C_{0}$ & -407.905 & -239.195 & -408.472 & -205.024 & -347.201 \\
\hline$C_{1}$ & 0.000 & 0.000 & 0.000 & 0.000 & 0.000 \\
\hline$C_{2}$ & -1.272 & -4.258 & -5.821 & -3.650 & -4.948 \\
\hline$C_{3}$ & 53.132 & 31.575 & 57.355 & 27.064 & 48.752 \\
\hline$C_{4}$ & -1.755 & -1.504 & -1.425 & -1.289 & -1.212 \\
\hline$C_{5}$ & 2.107 & 4.400 & 1.614 & 3.772 & 1.372 \\
\hline$C_{6}$ & 0.000 & 0.000 & 0.000 & 0.000 & 0.000 \\
\hline \multirow[t]{2}{*}{$C_{7}$} & 0.000 & 0.000 & 0.000 & 0.000 & 0.000 \\
\hline & D & \multicolumn{4}{|c|}{ ML and least-squares } \\
\hline$C_{0}$ & -407.905 & -412.142 & -438.344 & -353.264 & -296.642 \\
\hline$C_{1}$ & 0.000 & 0.000 & 0.000 & 0.000 & 0.000 \\
\hline$C_{2}$ & -1.272 & 0.000 & -4.673 & 0.000 & -6.927 \\
\hline$C_{3}$ & 53.132 & 52.505 & 60.590 & 45.004 & 43.308 \\
\hline$C_{4}$ & -1.755 & -2.193 & -1.426 & -1.879 & -1.060 \\
\hline$C_{5}$ & 2.107 & 2.512 & 1.803 & 2.153 & 1.400 \\
\hline$C_{6}$ & 0.000 & 0.000 & 0.000 & 0.000 & 0.000 \\
\hline \multirow[t]{2}{*}{$C_{7}$} & 0.000 & 0.000 & 0.000 & 0.000 & 0.000 \\
\hline & & \multicolumn{4}{|c|}{ Constrained estimates } \\
\hline$C_{0}$ & -407.905 & -420.957 & -410.061 & -360.820 & -353.680 \\
\hline$C_{1}$ & 0.000 & 0.000 & 0.000 & 0.000 & 0.000 \\
\hline$C_{2}$ & -1.272 & -2.779 & -0.305 & -2.382 & -0.263 \\
\hline$C_{3}$ & 53.132 & 56.300 & 52.586 & 48.257 & 45.355 \\
\hline$C_{4}$ & -1.755 & -1.621 & -1.812 & -1.389 & -1.563 \\
\hline$C_{5}$ & 2.107 & 1.988 & 2.156 & 1.704 & 1.860 \\
\hline$C_{6}$ & 0.000 & 0.000 & 0.000 & 0.000 & 0.000 \\
\hline$C_{7}$ & 0.000 & 0.000 & 0.000 & 0.000 & 0.000 \\
\hline
\end{tabular}

${ }^{*}$ In these cases, no run-outs occurred and the estimates are therefore the same.

maximum likelihood. Finally, we have presented two examples to illustrate the exponential model derived as well as to demonstrate the two methods of estimation proposed here.

Moreover, it is also important to mention the following points:

1. The method will enable one to extrapolate laboratory results obtained from any combination of values of $\sigma_{\min }$ or $\sigma_{\max }$ to any possible combination of values of $\sigma_{\min }$ or $\sigma_{\max }$ arising in a practical case; 
Table 3: True and estimated quartiles, based on the two samples in Table 1, with and without run-outs.

\begin{tabular}{|c|c|c|c|c|c|c|}
\hline \multirow[t]{2}{*}{ Condition } & \multirow[t]{2}{*}{ Quartile } & \multirow[t]{2}{*}{ True value } & \multicolumn{2}{|c|}{$\begin{array}{l}\text { Estimated value }^{1} \\
(n=7) \quad(n=20)\end{array}$} & \multicolumn{2}{|c|}{$\begin{array}{l}\text { Estimated value } \\
(n=7) \quad(n=20)\end{array}$} \\
\hline & & & \multicolumn{4}{|c|}{ ML unconstrained estimates } \\
\hline$\sigma_{m}=510$ & $Q_{25}$ & 12.4342 & 12.5012 & 12.4661 & 12.5142 & 12.4799 \\
\hline \multirow[t]{3}{*}{$\sigma_{M}=1054$} & $Q_{50}$ & 12.5431 & 12.6108 & 12.5764 & 12.6420 & 12.6096 \\
\hline & $Q_{75}$ & 12.7292 & 12.7981 & 12.7649 & 12.8606 & 12.8314 \\
\hline & $Q_{90}$ & 12.9752 & 13.0458 & 13.0140 & 13.1495 & 13.1245 \\
\hline$\sigma_{m}=510$ & $Q_{25}$ & 10.0150 & 10.0197 & 10.0385 & 10.0310 & 10.0516 \\
\hline \multirow[t]{3}{*}{$\sigma_{M}=1190$} & $Q_{50}$ & 10.1169 & 10.1154 & 10.1432 & 10.1427 & 10.1748 \\
\hline & $Q_{75}$ & 10.2911 & 10.2791 & 10.3222 & 10.3337 & 10.3853 \\
\hline & $Q_{90}$ & 10.5213 & 10.4955 & 10.5587 & 10.5861 & 10.6636 \\
\hline$\sigma_{m}=714$ & $Q_{25}$ & 14.9139 & 14.9301 & 14.9484 & 14.9452 & 14.9643 \\
\hline \multirow[t]{3}{*}{$\sigma_{M}=1054$} & $Q_{50}$ & 15.0433 & 15.0571 & 15.0752 & 15.0932 & 15.1135 \\
\hline & $Q_{75}$ & 15.2645 & 15.2741 & 15.2920 & 15.3464 & 15.3685 \\
\hline & $Q_{90}$ & 15.5570 & 15.5609 & 15.5785 & 15.6810 & 15.7056 \\
\hline$\sigma_{m}=714$ & $Q_{25}$ & 11.8862 & 11.8938 & 11.8880 & 11.9085 & 11.8957 \\
\hline \multirow{4}{*}{$\sigma_{M}=1190$} & $Q_{50}$ & 12.0059 & 12.0181 & 12.0084 & 12.0536 & 12.0312 \\
\hline & $Q_{75}$ & 12.2104 & 12.2307 & 12.2141 & 12.3015 & 12.2598 \\
\hline & $Q_{90}$ & 12.4807 & 12.5117 & 12.4860 & 12.6294 & 12.5620 \\
\hline & & & \multicolumn{4}{|c|}{ ML and least-squares } \\
\hline$\sigma_{m}=510$ & $Q_{25}$ & 12.4342 & 12.3172 & 12.5789 & 12.3298 & 12.3338 \\
\hline \multirow[t]{3}{*}{$\sigma_{M}=1054$} & $Q_{50}$ & 12.5431 & 12.4235 & 12.6896 & 12.4538 & 12.4631 \\
\hline & $Q_{75}$ & 12.7292 & 12.6052 & 12.8790 & 12.6658 & 12.6841 \\
\hline & $Q_{90}$ & 12.9752 & 12.8455 & 13.1294 & 12.9461 & 13.9763 \\
\hline$\sigma_{m}=510$ & $Q_{25}$ & 10.0150 & 9.8585 & 9.9735 & 9.8701 & 10.1102 \\
\hline \multirow[t]{3}{*}{$\sigma_{M}=1190$} & $Q_{50}$ & 10.1169 & 9.9569 & 10.0781 & 9.9850 & 10.2328 \\
\hline & $Q_{75}$ & 10.2911 & 10.1252 & 10.2568 & 10.1813 & 10.4425 \\
\hline & $Q_{90}$ & 10.5213 & 10.3477 & 10.4930 & 10.4408 & 10.7197 \\
\hline$\sigma_{m}=714$ & $Q_{25}$ & 14.9139 & 15.2713 & 14.9710 & 15.2869 & 14.7548 \\
\hline \multirow[t]{3}{*}{$\sigma_{M}=1054$} & $Q_{50}$ & 15.0433 & 15.4031 & 15.0985 & 15.4407 & 15.9007 \\
\hline & $Q_{75}$ & 15.2645 & 15.6285 & 15.3165 & 15.7036 & 15.1501 \\
\hline & $Q_{90}$ & 15.5570 & 15.9263 & 15.6046 & 16.0511 & 15.4797 \\
\hline$\sigma_{m}=714$ & $Q_{25}$ & 11.8862 & 12.0097 & 11.8439 & 12.0239 & 12.1229 \\
\hline \multirow{4}{*}{$\sigma_{M}=1190$} & $Q_{50}$ & 12.0059 & 12.1297 & 12.9632 & 12.1638 & 12.2603 \\
\hline & $Q_{75}$ & 12.2104 & 12.3347 & 12.1671 & 12.4030 & 12.4954 \\
\hline & $Q_{90}$ & 12.4807 & 12.6057 & 12.4367 & 12.7192 & 12.8060 \\
\hline & & & \multicolumn{4}{|c|}{ Constrained estimates } \\
\hline$\sigma_{m}=510$ & $\overline{Q_{25}}$ & 12.4342 & 12.5005 & 12.4654 & 12.5133 & 12.4778 \\
\hline \multirow[t]{3}{*}{$\sigma_{M}=1054$} & $Q_{50}$ & 12.5431 & 12.6090 & 12.574 & 12.6399 & 12.6045 \\
\hline & $Q_{75}$ & 12.7292 & 12.7945 & 12.7616 & 12.8564 & 12.8212 \\
\hline & $Q_{90}$ & 12.9752 & 13.0398 & 13.0087 & 13.1425 & 13.1077 \\
\hline$\sigma_{m}=510$ & $Q_{25}$ & 10.0150 & 10.0240 & 10.0367 & 10.0361 & 10.0482 \\
\hline \multirow[t]{3}{*}{$\sigma_{M}=1190$} & $Q_{50}$ & 10.1169 & 10.1260 & 10.1388 & 10.1550 & 10.1666 \\
\hline & $Q_{75}$ & 10.2911 & 10.3003 & 10.3133 & 10.3583 & 10.3690 \\
\hline & $Q_{90}$ & 10.5213 & 10.5306 & 10.5441 & 10.6271 & 10.6365 \\
\hline$\sigma_{m}=714$ & $Q_{25}$ & 14.9139 & 14.9302 & 14.9513 & 14.9453 & 14.9661 \\
\hline \multirow[t]{3}{*}{$\sigma_{M}=1054$} & $Q_{50}$ & 15.0433 & 15.0573 & 15.0821 & 15.0935 & 15.1178 \\
\hline & $Q_{75}$ & 15.2645 & 15.2746 & 15.3058 & 15.3470 & 15.3771 \\
\hline & $Q_{90}$ & 15.5570 & 15.5618 & 15.6014 & 15.6820 & 15.7199 \\
\hline$\sigma_{m}=714$ & $Q_{25}$ & 11.8862 & 11.8894 & 11.8883 & 11.9034 & 11.9019 \\
\hline \multirow[t]{3}{*}{$\sigma_{M}=1190$} & $Q_{50}$ & 12.0059 & 12.0075 & 12.0089 & 12.0412 & 12.0418 \\
\hline & $Q_{75}$ & 12.2104 & 12.2095 & 12.2152 & 12.2768 & 12.2809 \\
\hline & $Q_{90}$ & 12.4807 & 12.4765 & 12.4878 & 12.5883 & 12.5970 \\
\hline
\end{tabular}

2. Testing strategies (test designs) suggested for producing enough data will enable the above extrapolation possible. This will result in a substantial reduction as compared to traditional testing 

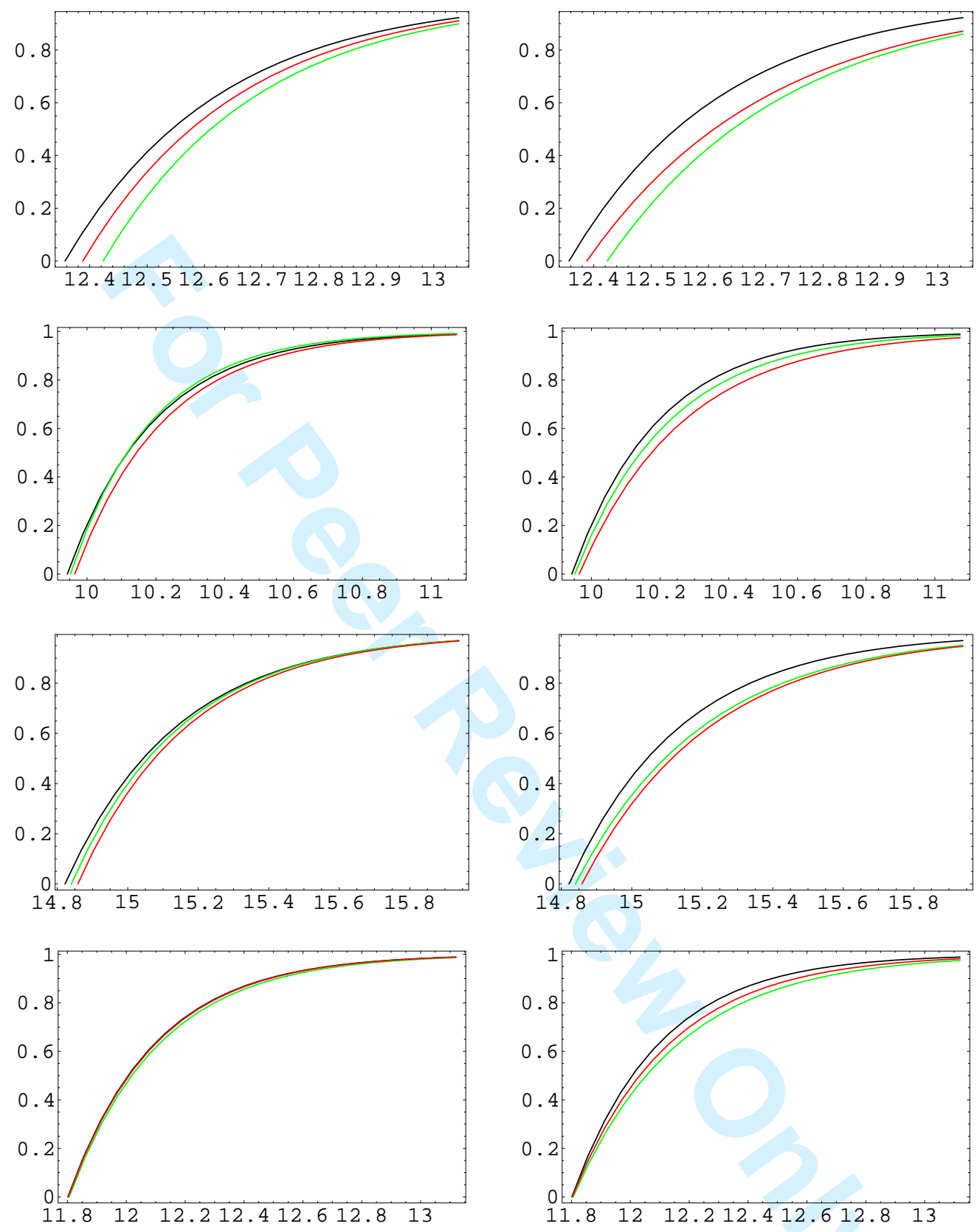

Figure 4: Cdf of lifetimes $\log N$ under the four conditions (from top to bottom: $\left(\sigma_{m}=510 ; \sigma_{M}=1054\right.$ ), $\left(\sigma_{m}=510 ; \sigma_{M}=1190\right),\left(\sigma_{m}=714 ; \sigma_{M}=1054\right)$ and $\left.\left(\sigma_{m}=714 ; \sigma_{M}=1190\right)\right)$. Estimated cdf based on the sample of size $n=7$ and $n=20$ are plotted in green and red, respectively, for the case without run-outs [figures in the left column (a)] and with run-outs [in the right column], for the unconstrained method. 
procedures; in fact, four levels are normally used in standard tests of constant $\sigma_{\min }$ or $\sigma_{\max }$;

3. Explicit simple formulas derived will facilitate the extrapolation (or interpolation) from lab results;

4. The normalization principle, together with the model proposed in this paper, would enable the analysis of damage accumulation when one knows the history of $\sigma_{\min }$ or $\sigma_{\max }$ as a function of time or cycle in a practical case;

5. The unconstrained and constrained methods of estimation proposed will facilitate the model fitting in a simple and efficient manner.

\section{Acknowledgements}

The authors are indebted to the Spanish Ministry of Science and Technology (Project BIA2005-07802C02-01) for partial support. The authors also express their thanks to a referee for making some suggestions which led to an improvement in the presentation of this paper.

\section{References}

ASTM (1981). Statistical Analysis of Fatigue Data, ASTM STP 744 (Eds., R.E. Little and J.C. Ekvall), The American Society for Testing and Materials.

Bastenaire F.A. (1972). New Method for the Statistical Evaluation of Constant Stress Amplitude Fatigue-Test Results, In Probabilistic Aspects of Fatigue, ASTM STP 511, 3-28.

Buckingham, E. (1914). On Physically Similar Systems: Illustrations of the Use of Dimensional Equations, Phys. Rev. 4, 345-376.

Buckingham, E. (1915a). The Principle of Similitude, Nature 96, 396-397.

Buckingham, E. (1915b). Model Experiments and the Form of Empirical Equations, Trans. ASME 37, 263-296.

Castillo E. (1988). Extreme Value Theory in Engineering, New York: Academic Press.

Castillo, E., Conejo, A., Pedregal, P., García, R. and Alguacil, N. (2001). Building and Solving Mathematical Programming Models in Engineering and Science, New York: John Wiley \& Sons.

Castillo, E. and Fernández-Canteli, A. (2001). A General Regression Model For Lifetime Evaluation and Prediction, International Journal of Fracture 107, 117-137.

Castillo, E. and Fernández-Canteli, A. (2006). A Parametric Lifetime Model for the Prediction of High Cycle Fatigue based on Stress Level and Amplitude, Fatigue and Fracture of Engineering Materials and Structures 29, 1031-1038.

Castillo E., Fernández-Canteli, A., Esslinger V. and Thürlimann, B. (1985). Statistical Model for Fatigue Analysis of Wires, Strands and Cables, IABSE P-82/85, 1-40.

Castillo, E., Fernández-Canteli, A. and Ruiz-Ripoll, M.L. (2006). A general model for fatigue damage due to any stress history, Int J Fatigue (2007), doi:10.1016/j.ijfatigue.2007.02.011.

Castillo E. and Galambos, J. (1987). Lifetime Regression Models Based on a Functional Equation of Physical Nature, Journal of Applied Probability 24, 160-169. 
Castillo E. and Hadi, A.S. (1995). Modelling Lifetime Data with Application to Fatigue Models, Journal of the American Statistical Association 90, 1041-1054.

Castillo, E., Hadi, A.S., Balakrishnan, N. and Sarabia, J. M. (2004). Extreme Value and Related Models with Applications in Engineering and Science, New York: John Wiley \& Sons.

Castillo, E., Hadi, A.S., Fernández-Canteli, A. and Conejo, A.J. (2004). A General Method for Local Sensitivity Analysis with Application to Regression Models and Other Optimization Problems, Technometrics 46, 430-444.

Castillo, E., Iglesias, A. and Ruiz-Cobo, R. (2004). Functional Equations in Applied Sciences, Amsterdam: Elsevier.

Castillo, E., López-Aenlle, M., Ramos, A., Fernández-Canteli, A., Kieselbach, R. and Esslinger, V. (2006). Specimen Length Effect on Parameter Estimation in Modelling Fatigue Strength by Weibull Distribution, International Journal of Fatigue, 1047-1058.

Castillo, E. and Ruiz-Cobo, R. (1992). Functional Equations in Science and Engineering, New York: Marcel Dekker.

Coleman, B.D. (1958). Statistics and Time Dependence of Mechanical Breakdown in Fibers, Journal of Applied Physics 29, 968-983.

Conejo, A.J., Castillo, E., Mínguez, R. and García-Bertrand, R. (2006). Decomposition Techniques in Mathematical Programming: Engineering and Science Applications, New York: Springer-Verlag.

Conway J.B. and Sjodahlo L.H. (1991). Analysis and representation of fatigue data, Cincinatti: ASM International.

Dowling N.E. and Thangjitham, S. (2000). An Overview and Discussion of Basic Methodology for Fatigue and Fracture Mechanics, ASTM STP 1389, pp. 3-36.

Fernández-Canteli, A. (1982). Statistical Interpretation of the Miner-Number Using an Index of Total Damage, IBASE Colloquium, Lausanne, pp. 309-319.

Galambos, J. (1987). The Asymptotic Theory of Extreme Order Statistics, Malabar, Florida: Krieger Publishing Co.

Pascual, F.G. and Meeker, W.Q. (1999). Estimating Fatigue Curves with the Random Fatigue-Limit Model, Technometrics 41, 277-302.

Spindel, J.E. and Haibach, E. (1981). Some Considerations in the Statistical Determination of the Shape of S-N Curves, In Statistical Analysis of Fatigue Data, ASTM STP 744 (Eds., R.E. Little and J.C. Ekvall), pp. 89-113, American Society for Testing and Materials. . 